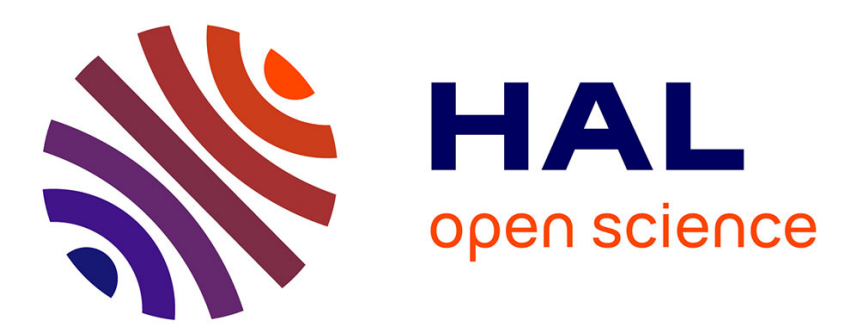

\title{
Transmission between chickens of an H7N1 Low Pathogenic Avian Influenza virus isolated during the epidemic of 1999 in Italy
}

J.L. Gonzales, J.A. van Der Goot, J.A. Stegeman, A.R.W. Elbers, G. Koch

\section{To cite this version:}

J.L. Gonzales, J.A. van Der Goot, J.A. Stegeman, A.R.W. Elbers, G. Koch. Transmission between chickens of an H7N1 Low Pathogenic Avian Influenza virus isolated during the epidemic of 1999 in Italy. Veterinary Microbiology, 2011, 152 (1-2), pp.187. 10.1016/j.vetmic.2011.04.022 hal-00719082

\section{HAL Id: hal-00719082 \\ https://hal.science/hal-00719082}

Submitted on 19 Jul 2012

HAL is a multi-disciplinary open access archive for the deposit and dissemination of scientific research documents, whether they are published or not. The documents may come from teaching and research institutions in France or abroad, or from public or private research centers.
L'archive ouverte pluridisciplinaire HAL, est destinée au dépôt et à la diffusion de documents scientifiques de niveau recherche, publiés ou non, émanant des établissements d'enseignement et de recherche français ou étrangers, des laboratoires publics ou privés. 


\section{Accepted Manuscript}

Title: Transmission between chickens of an H7N1 Low Pathogenic Avian Influenza virus isolated during the epidemic of 1999 in Italy

Authors: J.L. Gonzales, J.A. van der Goot, J.A. Stegeman,

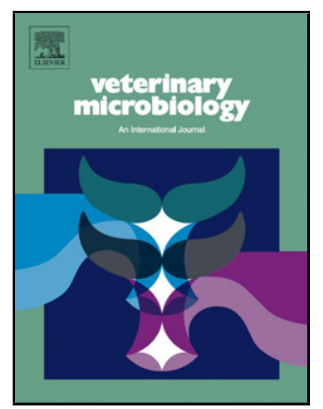
A.R.W. Elbers, G. Koch

PII: S0378-1135(11)00236-7

DOI: doi:10.1016/j.vetmic.2011.04.022

Reference: VETMIC 5285

To appear in: $\quad$ VETMIC

Received date: $\quad$ 4-1-2011

Revised date: 6-4-2011

Accepted date: $\quad$ 14-4-2011

Please cite this article as: Gonzales, J.L., van der Goot, J.A., Stegeman, J.A., Elbers, A.R.W., Koch, G., Transmission between chickens of an H7N1 Low Pathogenic Avian Influenza virus isolated during the epidemic of 1999 in Italy, Veterinary Microbiology (2010), doi:10.1016/j.vetmic.2011.04.022

This is a PDF file of an unedited manuscript that has been accepted for publication. As a service to our customers we are providing this early version of the manuscript. The manuscript will undergo copyediting, typesetting, and review of the resulting proof before it is published in its final form. Please note that during the production process errors may be discovered which could affect the content, and all legal disclaimers that apply to the journal pertain. 


\section{Transmission between chickens of an H7N1 Low Pathogenic}

2 Avian Influenza virus isolated during the epidemic of 1999

3 in Italy

4

5 J.L. Gonzales ${ }^{\text {a,b }}$, J.A. van $\operatorname{der} \operatorname{Goot}^{\mathrm{a}, \mathrm{c}}$, J.A. Stegeman ${ }^{\mathrm{b}}$, A.R.W. Elbers ${ }^{\mathrm{a}}$, G. Koch ${ }^{\mathrm{c}}$. 6

7

$8{ }^{\text {a }}$ Department of Epidemiology, Crisis organization and Diagnostics,

9 Central Veterinary Institute (CVI), part of Wageningen UR, P.O. Box 65, 8200 AB

10 Lelystad, The Netherlands.

$11{ }^{\mathrm{b}}$ Department of Farm Animal Health, Faculty of Veterinary Medicine, Utrecht

12 University P.O. Box 80, 151, 3508 TD Utrecht, The Netherlands.

$13{ }^{\mathrm{c}}$ Department of Virology, Central Veterinary Institute (CVI), part of Wageningen UR, 14 P.O. Box 65, 8200 AB Lelystad, The Netherlands.

15

16 Correspondence: Jose L Gonzales, Central Veterinary Institute (CVI), part of

17 Wageningen UR, P.O. Box 65, 8200 AB Lelystad, The Netherlands. Email:

$18 \quad$ jose.gonzales@wur.nl 


\section{Abstract}

The transmissibility of an H7N1 Low Pathogenic Avian Influenza (LPAI) virus isolated from a turkey flock during the large epidemic in Italy in 1999, was experimentally studied in chickens. Four group transmission experiments were performed. Infection and transmission were monitored by means of virus isolation on swab samples and antibody detection in serum samples. From the results of these groups, we estimated the mean infectious period at $7.7(6.7-8.7)$ days, the transmission rate parameter at $0.49(0.30-0.75)$ infections per infectious chicken per day and the basic reproduction ratio at $3.8(1.3-6.3)$. These estimates can be used for the development of surveillance and control programmes of LPAI in poultry.

Keywords: Avian Influenza, LPAI, H7N1, Transmission, Transmission parameters, Reproduction ratio

\section{Introduction}

Highly pathogenic Avian influenza (HPAI) is caused by AI viruses of $\mathrm{H} 7$ or H5 subtypes, that have evolved from preceding Low Pathogenic Avian Influenza viruses (LPAIv) (Banks et al., 2001; Capua and Maragon, 2000; DEFRA, 2008; Garcia et al., 1996). The H7N1 LPAI epidemic in Italy in 1999, which preceded the HPAI (1999 - 2000) epidemic, is one of the largest LPAI epidemics reported in a western country. In the absence of control measures - no compulsory regulations for control of LPAI outbreaks were in place - a total of 199 LPAI infected poultry farms were detected before the HPAI virus (HPAIv) took over (Busani et al., 2009; Capua and Maragon, 2000). Current knowledge does not allow to predict when an H5 or H7 LPAIv strain will mutate into a HPAIv strain. However, assuming that the molecular 
50

51

52

53

changes necessary for the virulence transformation occur at random, the probability that a LPAIv strain will mutate into a HPAIv strain will depend on the number of virus replicates, which in turn is associated with the number of birds acquiring infection. Hence, besides knowledge of the molecular biology of these viruses, quantitative knowledge of the transmission of LPAIv is important for the development of surveillance programmes that target early detection (see for example Graat et al., (2001)), hence reducing the probability of both undesired mutations and/or large epidemics.

To our knowledge, until now only one study quantifying LPAI virus transmission between chickens has been published in the peer reviewed literature - a chicken-derived H5N2 LPAI precursor virus of the 1983 HPAI epidemic in Pennsylvania (Van der Goot et al., 2003) - and there is no information about the transmissibility between chickens of H7 LPAIv subtypes . The objective of this study was to characterize - by means of transmission experiments - the betweenchicken transmission of an H7N1 LPAIv isolated from turkey during the 1999 epidemic in Italy.

\section{Material and methods}

\subsection{Virus and inoculation}

The LPAIv used for this study was the H7N1 LPAI A/turkey/Italy/1067/99 virus isolate. Its intravenous pathogenicity index and amino acid sequence of the hemagglutinin gene (GenBank accession number AF364134) have been published elsewhere (Banks et al., 2001; Capua and Maragon, 2000). Birds were inoculated both intranasally and intratracheally with $0.1 \mathrm{ml} /$ route of inoculum containing $10^{6}$ $\operatorname{EID}_{50}(50 \%$ egg infectious dose $) / \mathrm{ml}$. 
76

77

78

79

80

81

82

84

85

\subsection{Birds and experimental procedure}

A total of two transmission experiments were performed and each experiment consisted of two replicates (here referred to as trials). A trial comprised 10 six-weekold specific pathogen free (SPF) white leghorn chickens (Charles Rivers, The Netherlands). Five of the 10 birds were inoculated and the remaining five kept as contacts. The experiments were carried out in the High Containment Unit at the Central Veterinary Institute of Wageningen UR in Lelystad using biosafety level 3 procedures. Birds were housed in different pens in the same experimental room. Next to each experimental pen, 2 pairs of birds, housed in separate smaller pens, were placed as sentinels to detect whether between-pen (indirect) transmission should be considered and monitor independence of the trials. Chickens were housed 1 week before challenge to allow adaptation and during this period they were tested for presence of antibodies against influenza A virus using an in-house competitive NP ELISA (de Boer et al., 1990) and for virus by virus isolation. The day of inoculation $($ day $=0)$, contacts were removed from their pen and placed back 24 hours later. Trachea and cloaca swabs were collected daily from 1 to 10 days post inoculation (dpi) and then on 14, 17 and 21 dpi. Serum samples were taken at 7, 14 and 21 dpi. All surviving birds were euthanized at 21dpi. All experiments complied with the Dutch Law on Animal Experiments and were reviewed by an ethical committee. Trachea and cloaca swabs were used for virus isolation using SPF embryonated chicken eggs that were incubated for 9 days. Serum samples were tested for antibodies using the Hemagglutinin Inhibition test (HI) using 8 Hemagglutination units (HAU) of H7N1 as antigen. Diagnostic procedures were similar as those described by van der Goot et al. (2003). A bird was considered infected when it 
100

101

102

103

104

105

106

107

108

109

110

111

112

113

114

115

116

\section{3. Results}

118

119

120

121

123

scored positive in virus isolation or serology. A bird was considered infectious for the time it remained positive in virus isolation.

\subsection{Data analysis}

The basic reproduction ratio $\left(\mathrm{R}_{0}\right)$, which is defined as the average number of new infections caused by one infectious individual in a susceptible population, was estimated by the final size (FS) and the generalized linear model (GLM) methods as described by van der Goot et al. $(2003 ; 2005)$. The latent period was estimated as the time (in days) from inoculation to the first day the inoculated chicken was positive to virus isolation. The infectious period was estimated by fitting a parametric survival regression model with a Weibull distribution. In some cases, infected birds were detected positive by virus isolation, late, for example at $10 \mathrm{dpi}$ and were tested again at 14 dpi when they became negative and assumed to have recovered (Table 1). These cases were treated as interval censoring in the survival analysis. Survival analysis and the GLM method were carried out with the statistical software R (R Development Core Team, 2005). 

length of the infectious period (IP) was 7.7 (95\% Confidence Interval (CI): 6.7 - 8.7) days and the mean estimate of the transmission parameter $\beta$ was equal to 0.49 (95\%

\section{Discussion}

Herein the transmissibility in chickens of the H7N1 LPAIv, which caused a severe epidemic in poultry in Italy in 1999 and later evolved into a HPAI epidemic, was characterized. We have, in particular, estimated $\beta$ and the IP. These parameters give an indication of how fast the virus would spread to another susceptible bird and how long an infected chicken would remain infectious. Due to, mainly, the subclinical presentation of LPAI infections, it is difficult to accurately follow the course of the infection in field conditions, and characterization of transmission in the field would be limited to estimates of the $\mathrm{R}_{0}$ (Comin et al., 2010). Our experimental approach allowed us to follow the course of the infection under controlled conditions and have objective estimates of the transmission parameters. higher than those observed for an H5N2 LPAIv (van der Goot et al., 2003). This is

143 remarkable because the latter strain was isolated from a chicken whereas our strain

144 originated from turkey. Upon introduction, the virus here studied would reach its peak

145 (highest prevalence of infectious animals) faster and at a higher prevalence than the

146 H5N2 virus. Thus, it could be hypothesized that the infectivity of the flock would be

147 higher, resulting in a higher probability of transmission (assuming similar contact

148 rate) to other flocks. In this scenario, surveillance programmes would need to sample 
149

150

151

152

153

154

155

156

157

158

159

160

161

162

163

164

165

166

167

168

at a higher frequency if the objective is to detect in an early stage infection and to reduce the probability of between- flock transmissions.

Of the two methods used to estimate $\mathrm{R}_{0}$, the GLM method yielded a more

precise estimate (Table 2). This is because the GLM method uses all the detailed (day to day) information about the time course of the infection chain, while the FS method uses only the total number of animals that got infected throughout the experiment. We also used the FS method, because with that method there is no dependency on whether or not there is a latency period, as intrinsically assumed in the GLM method.

In conclusion, this study revealed valuable information on transmission parameters for LPAI virus in chickens. Such information can be used to improve or develop surveillance programmes and control measures.

\section{Acknowledgement}

The research was financially supported by EU contract QLK2-CT-2002-01454

AVIFLU and the Dutch Ministry of Economic Affairs, Agriculture and Innovation.

We thank Giovanni Cattoli (Instituto Zooprofilattico Sperimentale delle Venezie) for providing the virus for this study. 
168

169

170

171

172

173

174

175

176

177

178

179

180

181

182

183

184

185

186

187

188

189

190

191

192

\section{References}

Banks, J., Speidel, E.S., Moore, E., Plowright, L., Piccirillo, A., Capua, I., Cordioli, P., Fioretti, A., Alexander, D.J., 2001, Changes in the haemagglutinin and the neuraminidase genes prior to the emergence of highly pathogenic H7N1 avian influenza viruses in Italy. Archives of Virology 146, 963-973.

Busani, L., Valsecchi, M.G., Rossi, E., Toson, M., Ferrè, N., Pozza, M.D., Marangon, S., 2009, Risk factors for highly pathogenic H7N1 avian influenza virus infection in poultry during the 1999-2000 epidemic in Italy. The Veterinary Journal 181, 171-177.

Capua, I., Maragon, S., 2000, The avian influenza epidemic in Italy, 1999-2000. Avian Pathol 29, 289-294.

Comin, A., Klinkenberg, D., Stegeman, A., Busani, L., Marangon, S., 2010. Estimate of basic reproduction number $\left(\mathrm{R}_{0}\right)$ of low pathogenicity avian influenza outbreaks using a bayesian approach. In: Meeting of the Society for Veterinary Epidemiology and Preventive Medicine, Nantes, France, pp. 145 153.

de Boer, G.F., Back, W., Osterhaus, A.D.M.E., 1990, An ELISA for detection of antibodies against influenza A nucleoprotein in humans and various animal species. Archives of Virology 115, 47-61.

DEFRA 2008. Highly pathogenic avian influenza - H7N7, Oxfordshire, June 2008. Situation at $12.30 \mathrm{pm}$ Wednesday 2nd July.

Garcia, M., Crawford, J.M., Latimer, J.W., Rivera-Cruz, E., Perdue, M.L., 1996, Heterogeneity in the haemagglutinin gene and emergence of the highly pathogenic phenotype among recent $\mathrm{H} 5 \mathrm{~N} 2$ avian influenza viruses from Mexico. J Gen Virol 77, 1493-1504. 
193 Graat, E.A.M., de Jong, M.C.M., Frankena, K., Franken, P., 2001, Modelling the

194 effect of surveillance programmes on spread of bovine herpesvirus 1 between

195 certified cattle herds. Veterinary Microbiology 79, 193-208.

196 R Development Core Team 2005. R: A Language and Evironment for Statistical

197 Computing. ( Vienna, Austria: Foundation for Statistical Computing, 2005).

198 van der Goot, J.A., De Jong, M.C.M., Koch, G., van Boven, M., 2003, Comparison of

199 the transmission characteristics of low and high pathogenicity avian influenza

$200 \quad$ A virus (H5N2). Epidemiology and Infection 131, 1003-1013.

201 van der Goot, J.A., Koch, G., de Jong, M.C.M., van Boven, M., 2005, Quantification

202 of the effect of vaccination on transmission of avian influenza (H7N7) in

203 chickens PNAS 102 18141-18146.

204 
Table 1. Overview of the transmission process in the four group-transmission trials

\begin{tabular}{lccccccccccccc}
\hline Table 1a & \multicolumn{10}{c}{ Days post - inoculation } & \multicolumn{1}{c}{ Serology } \\
\cline { 2 - 11 } Trial 1 & 1 & 2 & 3 & 4 & 5 & 6 & 7 & 10 & 14 & 17 & 21 & \\
\hline Inoculated & $+\left.\right|^{\mathrm{a}}$ & $+\mid-$ & $+\mid-$ & $+\mid+$ & $+\mid+$ & $+\mid-$ & - & - & nd & nd & nd & $+(7)^{\mathrm{c}}$ \\
Inoculated & $+\mid-$ & $+\mid+$ & $+\mid+$ & $+\mid+$ & $+\mid+$ & $-\mid+$ & $-\mid+$ & - & nd & nd & nd & $+(7)$ \\
Inoculated & $+\mid+$ & $+\mid+$ & $-\mid+$ & $-\mid+$ & $-\mid+$ & $-\mid+$ & $-\mid+$ & - & nd & nd & nd & $+(6)$ \\
Inoculated & $+\mid-$ & $+\mid-$ & $+\mid+$ & $+\mid+$ & $-\mid+$ & $-\mid+$ & $-\mid+$ & - & nd & nd & nd & $+(7)$ \\
Inoculated & $+\mid-$ & $+\mid-$ & $+\mid-$ & $+\mid$ & - & - & - & - & nd & nd & nd & $+(4)$ \\
Contact & nd & - & $+\mid-$ & $+\mid-$ & $+\mid+$ & $+\mid+$ & $+\mid+$ & - & - & - & - & $+(7)$ \\
Contact & nd & $-\mid+$ & - & - & - & $+\mid-$ & $+\mid-$ & - & - & - & - & $-(2)$ \\
Contact & nd & - & - & - & - & - & - & $+\mid-$ & - & - & - & $+(5)$ \\
Contact & nd & - & - & - & - & - & - & - & $-\mid+$ & - & - & $+(6)$ \\
Contact & nd & - & - & - & - & - & - & - & - & - & - & - \\
\hline
\end{tabular}

\begin{tabular}{|c|c|c|c|c|c|c|c|c|c|c|c|c|}
\hline \multirow{2}{*}{$\begin{array}{l}\text { Table 1b } \\
\text { Trial } 2\end{array}$} & \multicolumn{11}{|c|}{ Days post - inoculation } & \multirow{2}{*}{ Serology } \\
\hline & 1 & 2 & 3 & 4 & 5 & 6 & 7 & 10 & 14 & 17 & 21 & \\
\hline Inoculated & $+\mid-$ & $-1+$ & $+\mid-$ & $+\mid-$ & - & - & - & 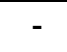 & nd & nd & nd & $+(8)$ \\
\hline Inoculated & $+\mid$ & $+\mid+$ & +++ & ++ & ++ & $-1+$ & - & & nd & nd & nd & $+(6)$ \\
\hline Inoculated & $+\mid-$ & $+\mid-$ & $+\mid-$ & $+\mid-$ & $+\mid-$ & $-1+$ & - & & nd & nd & nd & $+(5)$ \\
\hline Inoculated & ++ & ++ & ++ & ++ & ++ & $-1+$ & $-1+$ & & nd & nd & nd & $+(6)$ \\
\hline Inoculated & ++ & $+\mid-$ & $+\mid-$ & $+\mid-$ & ++ & $+\mid+$ & & & nd & nd & nd & $+(6)$ \\
\hline Contact & nd & - & - & - & - & $+\mid-$ & $+1+$ & - & - & - & - & - \\
\hline Contact & nd & - & - & $+\mid-$ & ++ & ++ & ++ & ++ & - & $-1+$ & - & $+(6)$ \\
\hline Contact & nd & - & - & - & - & $+1-$ & $+1-$ & $+\mid$ & - & - & - & $+(5)$ \\
\hline Contact & nd & - & - & - & - & & $+1+$ & $-1+$ & $-1+$ & - & - & $+(5)$ \\
\hline Contact & nd & - & - & - & - & $+1+$ & $+1+$ & $-1+$ & - & - & - & $+(6)$ \\
\hline
\end{tabular}

\begin{tabular}{|c|c|c|c|c|c|c|c|c|c|c|c|c|c|c|}
\hline \multirow{2}{*}{$\begin{array}{l}\text { Table 1c } \\
\text { Trial } 3\end{array}$} & \multicolumn{13}{|c|}{ Days post - inoculation } & \multirow{2}{*}{ Serolog } \\
\hline & 1 & 2 & 3 & 4 & 5 & 6 & 7 & 8 & 9 & 10 & 14 & 17 & 21 & \\
\hline Inoculated & $+\mid-$ & ++ & ++ & $+\mid-$ & - & ++ & - & - & - & - & - & - & - & $+(6)$ \\
\hline Inoculated & ++ & ++ & r & ++- & $+\mid-$ & ++ & - & - & - & - & - & - & - & $+(3)$ \\
\hline Inoculated & ++ & ++ & $+\mid-$ & +- & & ++ & - & - & - & - & - & - & - & $+(5)$ \\
\hline Inoculated & ++ & ++ & ++ & ++ & $-1+$ & $-\mid+$ & $-\mid+$ & $-\mid+$ & - & - & - & - & - & $+(7)$ \\
\hline Inoculated & $+\mid-$ & $+\mid-$ & ++ & $+1-$ & $+\mid-$ & ++ & $-1+$ & $-1+$ & $-1+$ & $-1+$ & - & - & - & $+(5)$ \\
\hline Contact & nd & & & $+1-$ & ++ & - & - & $+\mid-$ & - & - & - & - & - & $+(5)$ \\
\hline Contact & nd & 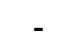 & $+1-$ & $+1-$ & $+1-$ & $+\mid+$ & $-1+$ & - & $-1+$ & - & - & - & - & $+(4)$ \\
\hline Contact & nd & & + & ++ & $-1+$ & $-1+$ & ++ & $-1+$ & $-1+$ & $-1+$ & - & - & - & $+(6)$ \\
\hline Contact & nd & & & $+1-$ & $+\mid-$ & $+\mid-$ & $+\mid-$ & $+\mid-$ & $-1+$ & $-1+$ & $-1+$ & - & - & $+(7)$ \\
\hline Contact & nd & + & & $+1-$ & & $+\mid-$ & - & $+\mid-$ & - & - & r & - & - & $-(2)$ \\
\hline
\end{tabular}

\begin{tabular}{|c|c|c|c|c|c|c|c|c|c|c|c|c|c|c|}
\hline \multirow{2}{*}{$\begin{array}{l}\text { Table 1d } \\
\text { Trial } 4\end{array}$} & \multicolumn{13}{|c|}{ Days post - inoculation } & \multirow{2}{*}{ Serology } \\
\hline & 1 & 2 & 3 & 4 & 5 & 6 & 7 & 8 & 9 & 10 & 14 & 17 & 21 & \\
\hline Inoculated & $+\mid-$ & $+\mid-$ & $+\mid-$ & +++ & +++ & $+\mid-$ & - & - & - & - & - & - & - & $+(7)$ \\
\hline Inoculated & $+\mid-$ & ++ & ++ & ++ & ++ & $+\mid+$ & $-1+$ & - & - & - & - & - & - & $+(7)$ \\
\hline Inoculated & $+\mid-$ & $+\mid-$ & $+\mid-$ & $+\mid-$ & $+\mid-$ & - & - & - & - & - & - & - & - & $+(6)$ \\
\hline Inoculated & $+\mid-$ & $+\mid+$ & $+\mid+$ & $+\mid+$ & $+\mid+$ & $-1+$ & $-1+$ & - & - & - & - & - & - & $+(7)$ \\
\hline Inoculated & $+\mid-$ & $+\mid-$ & $+\mid-$ & $+\mid-$ & $+\mid-$ & $-1+$ & $-1+$ & - & $-1+$ & - & - & - & - & $+(6)$ \\
\hline Contact & nd & - & - & - & $-1+$ & $+\mid+$ & $+\mid+$ & $-1+$ & $-1+$ & $-1+$ & $-1+$ & $-1+$ & - & $+(8)$ \\
\hline Contact & nd & - & $+\mid-$ & $+\mid-$ & $+\mid-$ & & $+\mid+$ & $-1+$ & $-1+$ & $-1+$ & - & $-1+$ & - & $+(6)$ \\
\hline Contact & nd & - & - & - & - & $-\mid+$ & $-1+$ & $-1+$ & $+\mid-$ & $-1+$ & - & - & - & $+(6)$ \\
\hline Contact & nd & - & $+\mid-$ & $+\mid-$ & - & - & $+\mid-$ & ++ & $-1+$ & $-1+$ & - & - & - & $+(6)$ \\
\hline Contact & nd & - & - & +++ & $-1+$ & $-\mid+$ & $-1+$ & $-1+$ & $-1+$ & $-1+$ & $-1+$ & - & - & $+(6)$ \\
\hline
\end{tabular}


${ }^{\mathrm{c}} \mathrm{HI}$ results at day 21 post-inoculation. Number in parenthesis are $\log _{2} \mathrm{HI}$ titers. Titers higher than 2 were considered positive. 
Table 2 Estimates (95\% confidence interval) of transmission parameters of H7N1 LPAI

\begin{tabular}{lc}
\hline Parameter & Estimates \\
\hline Number of contacts infected per trial & $4,5,5,5$ \\
Latent period & $\leq 1$ \\
Mean length Infectious period & $7.7(6.7-8.7)$ \\
Transmission rate $\beta\left(\right.$ day $\left.^{-1}\right)$ & $0.49(0.30-0.75)$ \\
Reproduction ratio $\mathrm{R}_{0}(\mathrm{GLM})$ & $3.8(1.3-6.3)$ \\
Reproduction ratio $\mathrm{R}_{0}(\mathrm{FS})^{\mathrm{a}}$ & $4.0(1.7-11.0)$ \\
${ }^{\mathrm{a}} \mathrm{R}_{0}$ estimates using the generalized lineal model (GLM) or the final size (FS) methods. \\
${ }^{\mathrm{b}}$ Inoculated birds were swab-sampled 24 hours post inoculation.
\end{tabular}

